\title{
Modelo funcional para la administración de una red de datos institucional
}

\author{
Fecha de recepción: 01/05/2020 • Fecha de aceptación: 28/05/2020 • Fecha de publicación:10/06/2020
}

Francisco Javier Galora Silva

Universidad Técnica de Ambato

Franciscog.s17@gmail.com

https://orcid.org/0000-0002-5464-5336

\section{RESUMEN}

El modelo de red de la UTA (Universidad Técnica de Ambato) indica que la política actual de la DITIC (Dirección de Tecnología de Información y Comunicación) con la que se encuentra administrada no ofrece la confiabilidad necesaria para el funcionamiento de cada uno de los elementos que la componen, es decir, que la planeación de la red aún no se rige en áreas de gestión definidas que puedan satisfacer requerimientos inmediatos y futuros, por ésta razón se debe tomar en cuenta las necesidades que involucren una adecuación en el sistema de transmisión de datos con la utilización de los recursos de administración disponibles. Mediante la monitorización de la red de datos de la DITIC de la UTA se ha realizado un análisis técnico de las áreas funcionales que actualmente otorgan servicios a los campus de Sur-Centro-Norte (Ingahurco - Huachi - Querochaca), lo que permite señalar las necesidades y requerimientos que presenta la red de datos, para establecer las condiciones más importantes que se deben tomar en cuenta para su correcta administración. Los requerimientos han sido presentados mediante áreas críticas de gestión, donde los requisitos expuestos obligan el planteamiento de normas reglamentarias que garanticen la operatividad entre los sistemas.

PALABRAS CLAVE: modelo funcional, red, transmisión, administración, eficiente, efectivo. 


\section{ABSTRACT}

The network model UTA (Technical University of Ambato) indicates that the current policy DITIC (Department of Information Technology and Communication) with which it is administered, does not offer the necessary reliability for operation of each of the its components, the network planning has not yet ruled on defined management areas that can meet immediate and future requirements, for this reason you should take into account the needs that involve an adjustment in the transmission system data with the use of management resources available. By monitoring network data DITIC UTA has made a technical analysis of the functional areas that currently provide services to the campus South-Centre-North (Ingahurco - Huachi - Querochaca), allowing identify needs and requirements that presents the network data to establish the most important conditions to be taken into account for proper administration. The requirements have been presented by critical management areas where exposed requirements oblige the approach of regulations to ensure the operability between systems.

KEYWORDS: functional model, network, transmission, administration, efficient, effective 


\section{Introducción}

Años atrás, a nivel mundial la gestión de los sistemas de datos estaba desarrollada y asignada a un operador, quien controlaba este sistema desde un determinado lugar.

En la actualidad son muchas las organizaciones que cuentan con un número considerable de ordenadores en operación y con frecuencia alejados unos de otros, éstos nuevos modelos de transmisión de datos obligan a cambiar su forma de administración, debido al desarrollo que presentan rigiéndose a nuevas tecnologías para exigir un funcionamiento eficiente y correcto de la red, lo que indica que un operador ya no puede ser de alta confiabilidad para controlar un sistema de transmisión de datos completamente (Vicente, C. A., 2003).

Estos modelos funcionales de red pueden ser diseñados para el manejo óptimo de un problema en particular. Previo a una consulta realizada a los administradores de red de la DITIC (Dirección de Tecnología de Información y Comunicación), es decir a su Director y al Administrador general de red, indican que en la UTA (Universidad Técnica de Ambato) no se tiene aún un modelo de manejo de fallas, seguridad, configuración, rendimiento y manejo de usuarios según un Estándar Internacional como la ISO (Organización de estándares Internacionales) o la ITU (Unión Internacional de Telecomunicaciones) los cuales promueven el desarrollo de la estandarización y las actividades a nivel mundial para facilitar el intercambio de servicios y bienes, derivándose de aquí el nombre de modelos funcionales (Galora Silva, F. J., 2015).

La administración del rendimiento aún no se centra en recolectar y analizar el tráfico que circula por la red de datos para determinar su comportamiento en tiempo real o en un intervalo de tiempo, así mismo la administración y localización de fallas no detectan una situación anormal que se pueda presentar y afectar el sistema de transmisión de datos (Vicente, C. A., 2003).

A nivel de facultades, dentro de la UTA el tratamiento de las redes denota falta de monitoreo, causando un problema al momento de notificar alguna falla. El modelo de red actual en la UTA debe ser analizado a partir de la arquitectura y topología que se encuentra actualmente establecida, indicando el lugar donde se pueda implementar una mejoría y haciendo énfasis en modelos de administración de redes según el modelo TMN (Red de gestión de las telecomunicaciones) de la ITU (Galora Silva, F. J., 2015), como el modelo OSI-NM (Gestión de la red) (U. I. de Telecomunicaciones., 2000); para definir una estructura organizacional con funciones bien definidas. En este contexto, este trabajo propone: establecer un modelo funcional para la administración de la red de Datos de la Universidad Técnica de Ambato, para realizar esto es necesario iniciar con un análisis de las características y el estado de funcionamiento de la red de datos actual, establecer políticas de administración de red dentro de un modelo funcional de gestión para mejorar el funcionamiento de la red de datos y demostrar el uso de las políticas establecidas para las áreas más críticas mediante la utilización de un software de simulación para cumplir con los requerimientos establecidos. 


\section{Metodología}

Para realizar un estudio estadístico en base a resultados obtenidos se considera la extracción de datos representativos de un conjunto respondiendo a cuantos, y cuáles son los sujetos necesarios para el análisis posterior, por consiguiente, las muestras extraídas de la población van a denotar una variabilidad entre los datos (Galindo, P. P. V., \& Vicente, M., 2012).

La aplicación utilizada para análisis de resultados: IBM SPSS Statistics Versión 21. IBM SPSS Statistics ofrece los procedimientos estadísticos principales que los analistas necesitan para tratar resultados de administración basados en investigación (IBM, 2014).

La propuesta de un modelo funcional para la administración de redes se basa en la recomendación de la ITU-T, el modelo TMN y en el modelo OSI-NM de ISO, donde se presenta una propuesta global que enfatiza en todos los aspectos relacionados a la buena operación de una red (Vicente, C. A., 2003).

Las políticas de administración de red son un conjunto de reglas que gobiernan la administración de los recursos de un sistema de datos, estas políticas tienen que ser congruentes con los objetivos de la institución y permitir el acceso a la información de una forma coherente, simple y segura (Montiel, N. A. N., 2005).

\section{Análisis de la red de Datos de la DITIC}

\subsection{Monitorización de la red de datos}

La política de monitoreo indica que por medio de la utilización de una herramienta de monitoreo en tiempo real (establecida cada 5 minutos) se establecen áreas de obtención de datos específicos para determinar un problema. La política de fallas muestra la generación de reportes del ancho de banda que está siendo utilizado por una facultad en un campus determinado, asimismo, originar un informe de conectividad por medio del envío de paquetes ICMP de solicitud y de respuesta (comando ping) hacia un switch o un servidor determinado para indicar el tiempo que tardan en comunicarse dos puntos remotos. La política del rendimiento, por su parte, determina la selección de un proceso de monitorización detallada a continuación: ubicarse en el lugar de un equipo específico para generar un informe relevante del mismo, por consiguiente, la generación de los informes respectivamente para ancho de banda y conectividad de equipos remotos que se realice cada 4 horas.

\subsection{Monitorización de ancho de banda}

Como se indica en la Figura 1, se tiene que:

a. Fuente de datos de entrada es el informe de los datos específicamente ubicados en los equipos administrados por la DITIC, esto indica el ancho de banda utilizado por la FISEI hacia Internet. En la FISEI, alrededor de las 07:40 am, están siendo enviados aproximadamente 60 megas 
hacia la DITIC (dirigido hacia Internet).

b. Fuente de datos de salida, evidencia el ancho de banda que se otorga a esta facultad, mismo que está siendo utilizado.

c. Política de solución de la DITIC: realizar una llamada vía telefónica al administrador de red de la FISEI para que solucione el problema que se presenta, debido a que es anormal la utilización de un exagerado ancho de banda en un intervalo determinado (entre las 07:35-07:45 am).

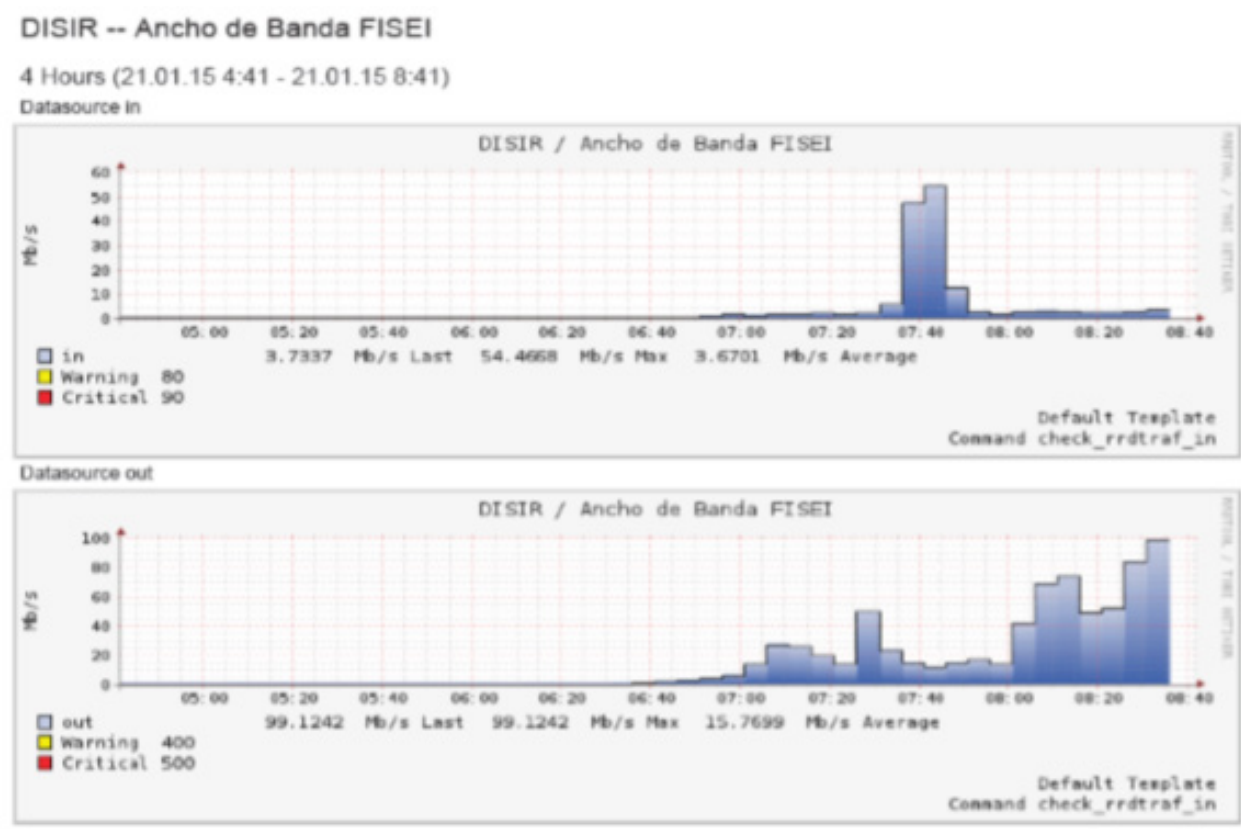

Figura 1. Ancho de banda utilizado por la Facultad de Ingeniería en Sistemas, Electrónica e Industrial

Fuente: DITIC

A nivel de Facultades dentro de la UTA el tratamiento de las redes denota falta de monitoreo, causando un problema al momento de notificar alguna falla.

\section{Informe de conectividad por medio del envío de paquetes ICMP}

Como se indica en la Figura 2, se tiene que:

a. Paquetes de solicitud y respuesta: indican el tiempo de respuesta en milisegundos, el presente envío de paquetes es hacia el servidor instalado en la facultad de Querochaca, de igual manera se la puede realizar a un equipo diferente desde el backbone de la DITIC. En Querochaca, alrededor de las 07:40 am en adelante, los retados (RTA) que presentan los paquetes de respuesta ICMP se establecen aproximadamente entre 0,5-1.5k (mil milisegundos), lo que indica que la conectividad no es óptima.

b. Paquetes perdidos: muestran el número de paquetes perdidos en aumento, dentro del intervalo de tiempo sin respuesta de conectividad ICMP. 
c. Política de solución de la DITIC: realizar una llamada vía telefónica al administrador de Querochaca para que solucione el problema que se presenta, debido a que, si existen tiempos de respuesta altos, o paquetes perdidos significa que el enlace está dando problemas, lo que puede depender de algún elemento que conforma el enlace, o la existencia de una sobrecarga. El retardo que presentan los paquetes de respuesta ICMP normalmente se estima hasta en 10 ms como valor pico, al momento en que se sobrepasa esta medida, se considera un enlace obsoleto.

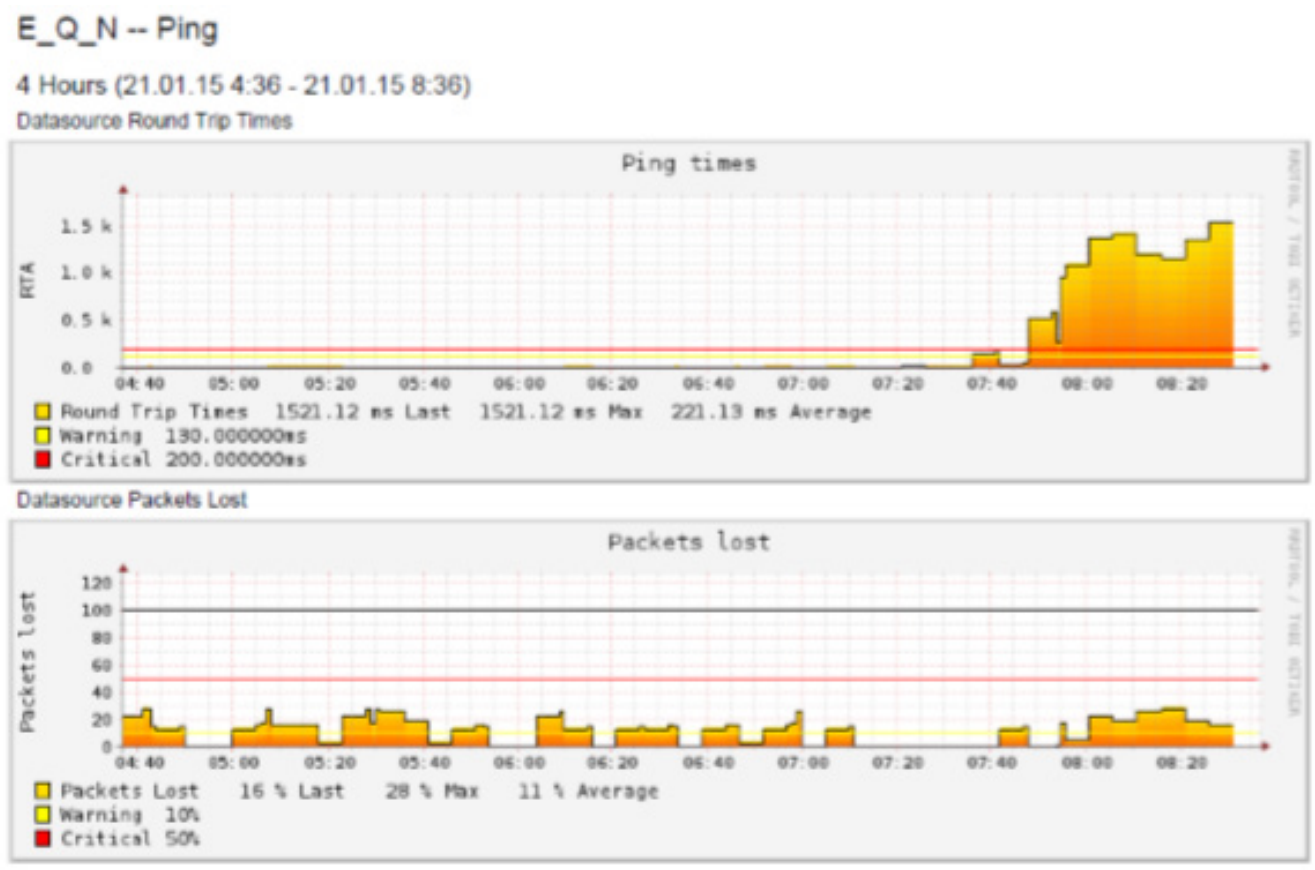

Figura 2. Informe de conectividad por medio del envío de paquetes ICMP de solicitud y de respuesta hacia el servidor de Querochaca (Zona Sur).

Fuente: DITIC

Por lo tanto, el establecimiento de políticas de administración de red dentro de éste modelo funcional de gestión que según Estándares Internacionales como la ISO (Organización de Estándares Internacionales), ITU (Unión Internacional de Telecomunicaciones), IEC (Comisión Electrotécnica Internacional), TIA (Asociación de la Industria de Telecomunicaciones), EIA (Asociación de Industrias Electrónicas) y el ANSI (Instituto Nacional Americano de Normalización), promueven el desarrollo de la estandarización y las actividades a nivel mundial para facilitar el intercambio de servicios y bienes, para mejorar el funcionamiento de la red de datos de la DITIC de la UTA. 


\section{3. Áreas críticas de gestión}

Tabla 1.

Áreas críticas de gestión

\begin{tabular}{|c|c|}
\hline \multirow[t]{2}{*}{ Áreas de gestión } & Modelo funcional para la administracion de la red de dtos de la DITIC \\
\hline & Punto critic \\
\hline Cableado Estructurado & $\begin{array}{l}\text { - Tendido de cable y colocación de canaletas sin consideraciones técnicas. } \\
\text { - No ha sido implementado bajo ningún estándar. } \\
\text { - Se realiza implementación de cableado estructurado sin presentación de un } \\
\text { anteproyecto. } \\
\text { - No se cuenta con una certificación de cableado estructurado. }\end{array}$ \\
\hline $\begin{array}{l}\text { Calidad de Energía } \\
\text { Eléctrica (aspectos } \\
\text { eléctricos) }\end{array}$ & $\begin{array}{l}\text { - Sistema de conexión a tierra. } \\
\text { - El cableado estructurado se encuentra cerca de las instalaciones eléctricas. } \\
\text { - No se da mantenimiento de las redes eléctricas que brinda energía a los } \\
\text { backbone. } \\
\text { - Existencia de cortocircuitos en los backbone. } \\
\text { - No se realiza gestión de alteraciones ambientales con influencia eléctrica. }\end{array}$ \\
\hline $\begin{array}{l}\text { Proyectos para } \\
\text { Mejoras/ Compras de } \\
\text { Equipos }\end{array}$ & $\begin{array}{l}\text { - No existe un modelo de presentación de proyectos para la implementación de un } \\
\text { servicio. } \\
\text { - No se tiene un modelo de caracterización técnica de equipos. } \\
\text { - Algunos softwares no cuentan con licencia. }\end{array}$ \\
\hline Inventario de Equipos & $\begin{array}{l}\text { - No existe un sistema de inventario personalizado para la red de datos de la } \\
\text { DITIC. }\end{array}$ \\
\hline $\begin{array}{l}\text { Administración de una } \\
\text { Red de Datos }\end{array}$ & $\begin{array}{l}\text { - Calidad de transmisión entre los campus Huachi-Ingahurco además de Huachi- } \\
\text { Querochaca y Huachi-Centro Cultural. } \\
\text { - No se tiene un control de las alteraciones que presentan los backbone. } \\
\text { - La demanda actual de usuarios es de } 2500 \text { equipos. - Servicio de utamático. } \\
\text { - No se realiza un control de acciones en la red de datos. } \\
\text { - Falta de implementación de políticas de administración dentro de un modelo } \\
\text { funcional para las áreas de gestión de la red de datos de la DITIC para la UTA. } \\
\text { - En algunas facultades no se cuenta con personal técnico para la administración } \\
\text { de la red. }\end{array}$ \\
\hline $\begin{array}{l}\text { Servicios de Red } \\
\text { (Servidor Proxy - } \\
\text { Redes Inalámbricas) }\end{array}$ & $\begin{array}{l}\text { - Utilización de una sola dirección IP para petición de ancho de banda desde las } \\
\text { facultades hacia el backbone de la DITIC. } \\
\text { - Saturación del servicio de Internet que brinda la red UTA WiFi. }\end{array}$ \\
\hline Sistemas de Monitoreo & $\begin{array}{l}\text { - El monitoreo hace énfasis en el ancho de banda que brinda a cada facultad. } \\
\text { - El software de monitoreo se actualiza cada } 3 \text { minutos. } \\
\text { - La monitorización que se realiza es visual. }\end{array}$ \\
\hline
\end{tabular}

Fuente: DITIC 


\section{Planteamiento de Políticas de Administración para la Red de Datos}

El planteamiento de políticas de administración para la red de datos de la DITIC para las áreas de gestión definidas se establece como normas reglamentarias para la arquitectura de la administración de red. Este modelo define una estructura organizacional con funciones bien definidas (Naranjo Villacrés, D. R., \& Ortega Tobar, P. E., 2006).

En referencia a las áreas de gestión, las políticas para la administración de la red de datos toman en cuenta: satisfacer los requerimientos inmediatos y futuros de la red en base a las necesidades que se presentan, planeación de reglas normalizadas dentro de un modelo de gestión, monitorización de información referente al comportamiento de la red, caracterización del tráfico, calidad de transmisión y recepción de información, administración de reportes, administración de la seguridad (detección de intrusos, políticas de seguridad) (Ruiz, et al, 2008).

\section{Resultados}

Los resultados obtenidos se analizarán en base a la estadística descriptiva, la cual desarrolla un conjunto de técnicas cuya finalidad es presentar y reducir diferentes datos observados entorno a un conjunto (Fernández, S. F., Sánchez, J. M. C., Córdoba, A., \& Largo, A. C., 2002).

\section{Herramienta Proxy}

A causa de que han sido definidas las áreas de gestión por intermedio de la DITIC, y siendo determinados los requerimientos en base al análisis de los puntos críticos técnicos reales, se establece que la DITIC de la UTA en relación a los puntos críticos que presenta el modelo de red actual constituye la implantación de políticas de administración en un servidor proxy (Tabla 2) como una opción para el manejo óptimo del mismo.

Tabla 2.

Características de servidor proxy

\begin{tabular}{ll}
\hline $\begin{array}{l}\text { Servidor Proxy (Proxy Server) } \\
\text { Descripción }\end{array}$ & Características \\
\hline Hardware & CPU Model: AMD E-300 APU Radeon \\
& (tm) HD Graphics \\
& CPU Mhz: 1300.000 \\
& CPU Cores: 2 \\
& Cache Size: 512 KB \\
\hline Disco Duro & Size: 50 G \\
\hline Memoria Ram & Total: $1,6 \mathrm{G}$
\end{tabular}

Fuente: elaboración propia 
En la Tabla 3 se evidencian las políticas de administración para el análisis de resultados con Proxy.

Tabla 3.

Políticas de administración para el análisis de resultados

\begin{tabular}{ll}
\hline Adquisición de datos estadísticos descriptivos \\
\hline Administración de Fallas & $\begin{array}{l}\text { Política de localización de una falla enfocada en la detección y aislamiento: } \\
\text { Interpretación de resultados del servidor proxy con y sin generación de carga }\end{array}$ \\
\hline $\begin{array}{l}\text { Administración del } \\
\text { Monitoreo }\end{array}$ & Statistics Versión 21. para interpretación de resultados \\
& - Política de análisis realizado según información de monitorización obtenida: Análisis \\
& estadístico descriptivo de los datos obtenidos \\
\hline
\end{tabular}

Fuente: Elaboración propia

Para la notificación de actividades del Servidor la aplicación utilizada para generar notificaciones del Servidor Proxy: Uptime Robot, una aplicación diseñada para ayudar a mantener los servidores funcionando, para lo cual se efectúa un seguimiento del mismo cada 5 minutos generando una alerta mediante el envío de un e-mail cuando no se encuentran en funcionamiento (B. B. T. U. S. T. L. Sti., 2014).

En la Figura 3 se muestra cómo es la configuración de la aplicación Uptime Robot

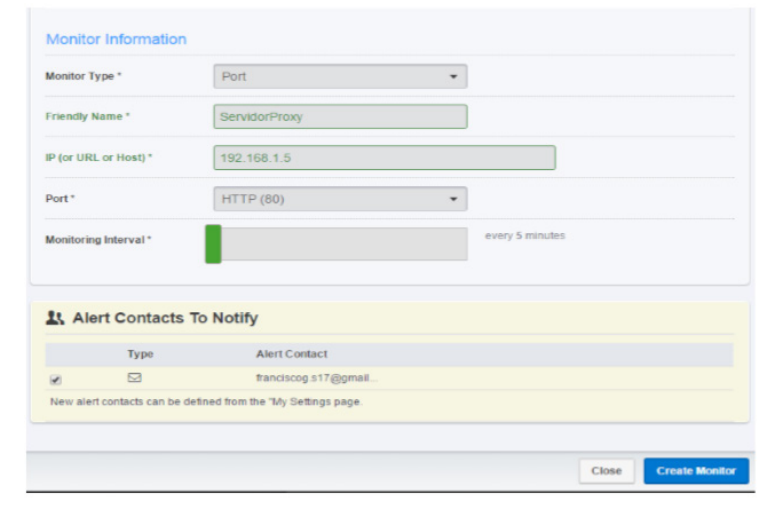

Figura 3. Configuración de la aplicación Uptime Robot

Fuente: elaboración propia

Uptime Robot permite generar una alerta mediante el envío de un e-mail hacia el administrador (Webmaster-franciscog.s17@gmail.com), en la cual indica el estado de funcionamiento del Servidor. Ver Figura 4.

La notificación de error del servidor proxy ha sido generada en el intervalo de tiempo en el cual se realizaron las pruebas de carga. 


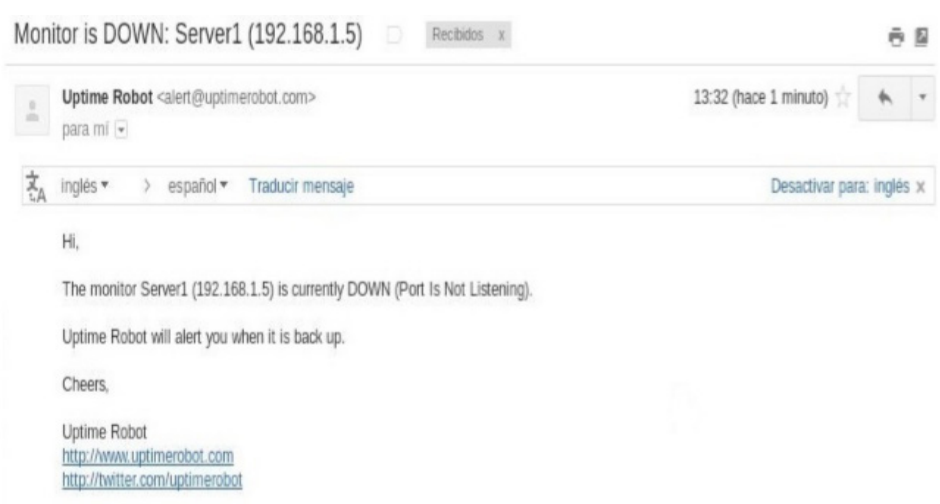

Figura 4. Generación de alerta mediante el envío de un e-mail hacia el administrador-franciscog.s17@gmail.com Fuente: elaboración propia.

\section{Las políticas de administración aplicadas para la aplicación mencionada se pueden ver en la Tabla 4.}

Tabla 4.

Políticas de administración aplicadas

\begin{tabular}{|c|c|}
\hline \multicolumn{2}{|c|}{ Políticas de administración aplicadas } \\
\hline Administración de Fallas & $\begin{array}{l}\text { Política de notificación de existencia de una falla y el lugar donde se ha generado: } \\
\text { Servidor Proxy esta inactivo (Server Proxy is currently Down) originado en la IP } \\
\text { 192.168.1.5 }\end{array}$ \\
\hline Administración de Reporte & $\begin{array}{l}\text { - Política de fallas notificadas por un sistema de alarmas: } \\
\text { Aplicación Uptime Robot para generar notificaciones } \\
\text { - Política de registro de reporte que enuncia recuperación de la red: } \\
\text { - Registro \#1: } \\
\text { Notificación número 1: Server is Down } \\
\text { - Recuperación de la red: Reinicio de la herramienta Squid: service squid restart }\end{array}$ \\
\hline $\begin{array}{l}\text { Administración de } \\
\text { Seguridad }\end{array}$ & $\begin{array}{l}\text { Política de detección de intrusos mediante reporte de eventos: Notificación del puerto } \\
\text { cuando no escucha (Port is not listening) }\end{array}$ \\
\hline $\begin{array}{l}\text { Administración del } \\
\text { monitoreo }\end{array}$ & $\begin{array}{l}\text { - Política de control de acceso periódico a la información de la red: } \\
\text { Monitorización del servidor proxy cada } 5 \text { minutos mediante notificación del puerto } \\
3128 \\
\text { - Política de mensajes enviados desde los recursos de red: } \\
\text { Generación de una alarma mediante el envío de un e-mail hacia el administrador } \\
\text { (franciscog.s17@gmail.com) }\end{array}$ \\
\hline
\end{tabular}

Fuente: Elaboración propia 
El comportamiento de los valores obtenidos ha sido analizado en base al estudio estadístico de variables $t$ de student, a continuación, las medidas de tendencia central con sus respectivas medidas de dispersión que indican el siguiente comportamiento para un servidor Proxy sin prueba de carga (Tabla 5).

Tabla 5.

Servidor Proxy

\begin{tabular}{|c|c|}
\hline \multicolumn{2}{|c|}{ Servidor Proxy sin prueba de carga } \\
\hline \multirow{3}{*}{$\begin{array}{l}\text { CPU (2 núcleos) Porcentaje } \\
\text { de CPU }\end{array}$} & Media Aritmética: 1,2645; Desviación Típica: 1,13467; Error Estándar: \\
\hline & 0,11347 \\
\hline & Rango de valores significativos del $95,5 \%$ de probabilidad: \\
\hline \multicolumn{2}{|l|}{ Utilizado } \\
\hline & Límite inferior: 1,0394 - Límite superior: 1,4896 \\
\hline \multirow[t]{2}{*}{$\begin{array}{l}\text { I/O RED (tps - cantidad de } \\
\text { transferencias u }\end{array}$} & Mediana: 0,0000 \\
\hline & Recorrido Intercuartilico: 2,78 \\
\hline \multirow{2}{*}{ operaciones por segundo) } & Media Aritmética: 0,9299; Desviación Típica: 0,29156; Error Estándar: \\
\hline & 0,2196 \\
\hline \multirow{3}{*}{$\begin{array}{l}\text { RAM (Gb de memoria } \\
\text { utilizada) Media Aritmética: }\end{array}$} & \\
\hline & Rango de valores significativos del $95,5 \%$ de probabilidad: \\
\hline & Límite inferior: 0,8720 - Límite superior: 0,9878 \\
\hline
\end{tabular}

Fuente: elaboración propia

a. CPU: El valor de la media aritmética 1,2645 \%, evidencia el porcentaje de utilización del CPU más representativo en el intervalo de datos muestrales. Con respecto al valor de la media aritmética, los valores muestrales en general tienden alejarse del promedio un 1,13467 \%. El error estándar de la media aritmética $0,11347 \%$, indica el valor estimado de cuánto puede variar la media aritmética con respecto a otras tomas muestrales de datos.

El rango de valores significativos que presentan los valores generados donde se encuentra la verdadera media aritmética con una fiabilidad del 95,5\%, se establece en el rango entre 1,0394 $\%$ y $1,4896 \%$.

b. I/O RED (Estadísticas de Entrada-Salida): El valor de la mediana muestra que el $50 \%$ de las muestras son mayores a 0,0000 tps y el otro $50 \%$ se mantienen en 0,0000 tps. El recorrido intercuartílico determina que los valores muestrales más representativos se encuentran alrededor de 2,78 tps.

C. RAM: El valor de la media aritmética 0,9299Gb indica el tamaño de memoria más representativo utilizada por el Servidor. Con respecto al valor de la media aritmética, los valores muestrales en general tienden alejarse del promedio 0,29156Gb. El error estándar de la media aritmética $0,2196 \mathrm{~Gb}$, indica el valor estimado de cuánto puede variar la media aritmética con 
respecto a otras tomas muestrales de datos. El rango de valores significativos que presenta los valores generados donde se encuentra la verdadera media aritmética con una fiabilidad del $95,5 \%$, se establece en el rango entre 0,8720Gb y 0,9878Gb.

Mientras que con el servidor Proxy con prueba de carga se muestra de la siguiente manera, ver Tabla 6.

Tabla 6.

Servidor Proxy

\begin{tabular}{|c|c|}
\hline \multicolumn{2}{|c|}{ Servidor Proxy con prueba de carga } \\
\hline & Media Aritmética: 9,177; Desviación Típica: 6,70746; Error Estándar: \\
\hline CPU (2 núcleos) Porcentaje & 0,29997 \\
\hline & Rango de valores significativos del $95,5 \%$ de probabilidad: \\
\hline \multicolumn{2}{|l|}{ Utilizado } \\
\hline & Límite inferior: 8,5813 - Límite superior: 9,7600 \\
\hline $\begin{array}{l}\text { I/O RED (tps - cantidad de } \\
\text { transferencias } u\end{array}$ & Mediana: 0,0000 \\
\hline operaciones por segundo) & Recorrido Intercuartilico: 16,36 \\
\hline \multirow{3}{*}{$\begin{array}{l}\text { RAM (Gb de memoria } \\
\text { utilizada) Media Aritmética: }\end{array}$} & $\begin{array}{l}\text { Media Aritmética: 0,9747; Desviación Típica: 0,49112; Error Estándar: } \\
0,2196\end{array}$ \\
\hline & Rango de valores significativos del $95,5 \%$ de probabilidad: \\
\hline & Límite inferior: 0,9615 - Límite superior: 1,0178 \\
\hline
\end{tabular}

Fuente: elaboración propia

a. CPU: El valor de la media aritmética $9,177 \%$, indica el porcentaje de utilización del CPU más representativo en el intervalo de datos muestrales. Con respecto al valor de la media aritmética, los valores muestrales en general tienden alejarse del promedio un 6,70746 \%. El error estándar de la media aritmética 0,29997 \%, muestra el valor estimado de cuánto puede variar la media aritmética con respecto a otras tomas muestrales de datos. El rango de valores significativos que presenta los valores generados donde se encuentra la verdadera media aritmética con una fiabilidad del $95,5 \%$, se establece en el rango entre 8,5813 \% y 9,7600 \%.

b. I/O RED (Estadísticas de Entrada-Salida): El valor de la mediana refleja que el $50 \%$ de las muestras son mayores a 0,0000 tps y el otro $50 \%$ se mantienen en 0,0000 tps. El recorrido intercuartílico determina que los valores muestrales más representativos se encuentran alrededor de 16,36 tps.

C. RAM: El valor de la media aritmética $0,9747 \mathrm{~Gb}$ refleja el tamaño de memoria más representativo utilizada por el Servidor. Con respecto al valor de la media aritmética, los valores 
muestrales en general tienden alejarse del promedio 0,49112Gb. El error estándar de la media aritmética $0,2196 \mathrm{~Gb}$, indica el valor estimado de cuánto puede variar la media aritmética con respecto a otras tomas muestrales de datos. El rango de valores significativos que presenta los valores generados donde se encuentra la verdadera media aritmética con una fiabilidad del $95,5 \%$, se establece en el rango entre $0,9615 \mathrm{~Gb}$ y $1,0178 \mathrm{~Gb}$.

Por su parte, en la Figura 5 se muestra la Topología de Red con el sistema operativo utilizado GNU/Linux CentOS 6.5. Política de administración de seguridad: Registro de acceso. Superusuario: root

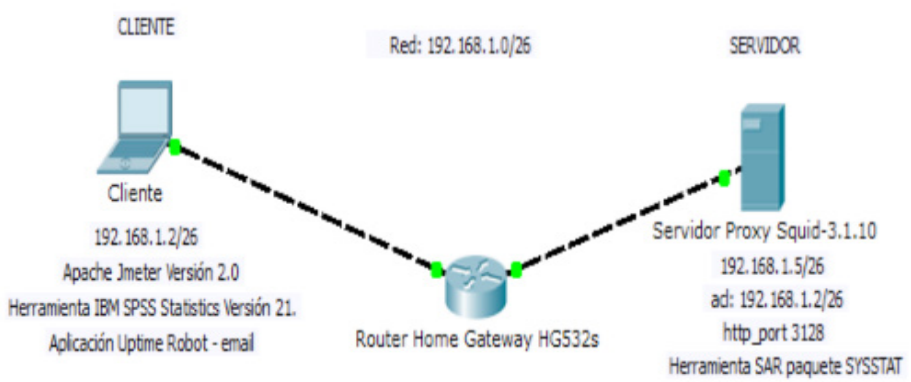

Figura 5. Topología de red

Fuente: elaboración propia

\section{Servidor Squid Versión 3.1.10}

El Servidor Intermediario utilizado Squid Versión 3.1.10. Squid muestra un alto desempeño utilizado entre los sistemas operativos como GNU/Linux (CentOS) (Dueñas, J. B., 2013). Es confiable, robusto y versátil debido a que consiste en un programa principal como servidor, utilizando algunas herramientas para la administración de los clientes. En la Tabla 7 se muestran las características de la herramienta Squid-3.1.10

Tabla 7.

Características de la herramienta Squid-3.1.10

\begin{tabular}{ll}
\hline Herramienta Squid 3.1.10 \\
\hline Prestaciones & - Funciona como Servidor Intermediario y caché de contenido de Red para los \\
& - HTTP, FTP, GOPHER y WAIS, Proxy de SSL, caché transparente, WWCP, \\
& aceleración HTTP, caché de consultas DNS \\
& - Capacidad de filtración de contenido \\
& - Implementación de control de acceso por IP y por usuario \\
\hline $\begin{array}{l}\text { Directorio de edición } \\
\text { de squid }\end{array}$ & /etc/squid/squid.conf visible_hostname: localhost.localdomain \\
\hline
\end{tabular}




\begin{tabular}{ll}
\hline Configuración & \\
\hline Control de acceso & $\begin{array}{l}\text { - Controla el tráfico de los clientes hacia Internet estableciendo Listas de Control de } \\
\text { Acceso que definan una red o bien ciertos anfitriones en particular. }\end{array}$ \\
& $\begin{array}{l}\text { - A cada lista se le asigna una Regla de Control de Acceso que permitirá o denegará } \\
\text { el acceso a Squid }\end{array}$ \\
\hline Opción http_port & Indicar el puerto a través del cual escuchará peticiones Squid \\
\hline Opción cache_dir & $\begin{array}{l}\text { Establece el tamaño que utiliza Squid para almacenamiento de caché en el disco } \\
\text { duro El formato de cache ufs puede llegar a bloquear el proceso principal de Squid } \\
\text { en operaciones de entrada/salida sobre el sistema de archivos cuando hay muchos } \\
\text { clientes conectados }\end{array}$ \\
\hline
\end{tabular}

Fuente: elaboración propia

Y en la Tabla 8 se explican las políticas de administración aplicadas del servidor mencionado.

Tabla 8.

Políticas de administración aplicadas

Políticas de administración aplicadas en herramienta SQUID-3.1.10

Administración de

1. Política de control de acceso: Implementación de una lista de control de acceso acl

seguridad localnet src 192.168.1.2 acl nourl url_regex "etc/squid/nourl" Implementación de una regla de control de acceso http_access allow localnet http_access deny localnet nourl

2. Política de acceso restrictivo: Implementación de una lista de control con acceso restrictivo a páginas web Directorio: etc/squid/nourl www.xxx.com xxx.com xxx www. porno.com porno.com porno

\begin{tabular}{ll}
\hline Puerto TCP/IP & http_port 3128 \\
\hline $\begin{array}{l}\text { Políticas de Administración } \\
\text { de Squid }\end{array}$ & - Se utiliza el formato aufs, que emplea el mismo formato de ufs, funcionando de \\
& $\begin{array}{l}\text { manera asincrónica para conseguir un mejor desempeño con muchos clientes } \\
\text { conectados. }\end{array}$ \\
& - Se utiliza el formato aufs para crear en el directorio /var/spool/squid un caché de \\
& $100 \mathrm{MB}$, dividido en jerarquías de 16 directorios subordinados, hasta 256 niveles \\
& cada uno: cache_dir aufs /var/spool/squid 10016256 \\
\hline Opción http_port & Indicar el puerto a través del cual escuchará peticiones Squid \\
\hline
\end{tabular}

Fuente: elaboración propia

\section{La Figura 6 presentada muestra la prueba de carga del Servidor Proxy Squid-3.1.10}




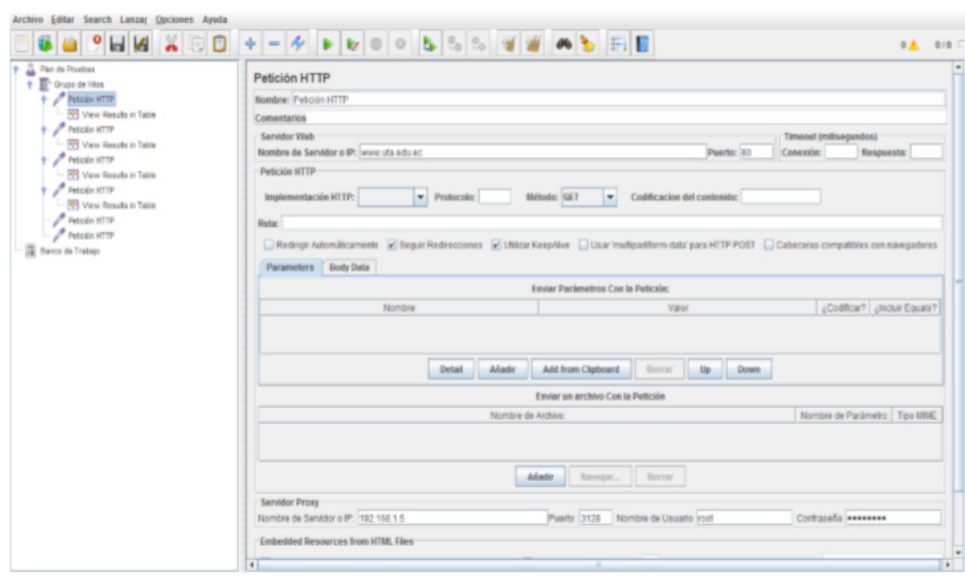

Figura 6. Prueba de carga del servidor Proxy Squid-3.1.10

Fuente: elaboración propia

\section{Herramienta Apache JMeter 2.0}

La aplicación de escritorio Apache JMeter (ver Tabla 9) es un software de código abierto que está desarrollado para representar una carga pesada en un servidor, grupo de servidores, la red o el objeto para probar su resistencia o para analizar el rendimiento general bajo diferentes tipos de carga (A. S. Foundation., 2013).

Tabla 9.

Herramienta Apache JMeter 2.0

\begin{tabular}{|c|c|}
\hline \multicolumn{2}{|c|}{ Configuración del plan de pruebas Política de administración de fallas } \\
\hline $\begin{array}{l}\text { Política de prueba } \\
\text { de diagnóstico para } \\
\text { localización de una falla }\end{array}$ & Grupos de hilos 2000 - Contador de bucle sin fin \\
\hline $\begin{array}{l}\text { - Política de } \\
\text { establecimiento de } \\
\text { información de gestión a } \\
\text { monitorizar }\end{array}$ & $\begin{array}{l}\text { Muestreador: } \\
\text { Peticiones HTTP - Puerto } 80 \\
\text { Servidor IP: } 192.168 .1 .5 \text { - Puerto: } 3128\end{array}$ \\
\hline $\begin{array}{l}\text { - Política de análisis de } \\
\text { protocolo para determinar } \\
\text { el tráfico fuera de un } \\
\text { patrón definido }\end{array}$ & Usuario: root \\
\hline $\begin{array}{l}\text { Política de sondeo y event } \\
\text { reporting (Servidor Proxy) }\end{array}$ & Receptor Ver resultados en tabla \\
\hline $\begin{array}{l}\text { Servidores Web } \\
\text { configurados }\end{array}$ & Configurar peticiones http mediante accesos de páginas Web \\
\hline
\end{tabular}

Fuente: elaboración propia 


\section{Herramienta SAR}

Mientras que SAR es una herramienta que significa informe de actividad del sistema, la cual en CentOS se encuentra incluida en el paquete de sysstat (IBM., 2014). La utilización de la CPU, intercambio de memoria, y las estadísticas de transferencia de red son parte de la generación de datos que permite sysstat, asimismo los informes originados en base al rendimiento pueden ser utilizados para localizar cuellos de botella y múltiples estadísticas de los servidores. SAR recopila, anuncia, y guarda la información de la actividad del sistema para que pueda ser visualizada (ver Tabla 10).

Tabla 10.

Herramienta SAR

\begin{tabular}{|c|c|}
\hline \multicolumn{2}{|c|}{ Configuración del comando SAR en el servidor proxy } \\
\hline $\begin{array}{l}\text { Directorio de edición: } \\
\text { vi /etc/cron.d/sysstat }\end{array}$ & $\begin{array}{l}* / 10 * * * * \text { root } / \text { usr } / \text { lib64/sa/sa1 - - - d } 11 \text { \# generate a daily summary of process } \\
\text { accounting at } 23: 535323 * * * \text { root /usr/lib64/sa/sa2 -A }\end{array}$ \\
\hline $\begin{array}{l}\text { Política de } \\
\text { establecimiento }\end{array}$ & CPU sar -u 150 \\
\hline $\begin{array}{l}\text { de mecanismos } \\
\text { de monitorización }\end{array}$ & I/O RED (Estadísticas de Entrada/Salida) sar -b 150 \\
\hline $\begin{array}{l}\text { Política de obtención } \\
\text { de información a } \\
\text { monitorizar }\end{array}$ & RAM sar -r 150 \\
\hline $\begin{array}{l}\text { Política de vigilancia } \\
\text { y administración de } \\
\text { respuesta de red }\end{array}$ & $\begin{array}{l}\text { Respuesta Obtención de resultados en código plano Directorio de generación: } \\
\text { LC_ALL=C sar (parámetro) >/tmp/sar.data.txt }\end{array}$ \\
\hline
\end{tabular}

Fuente: elaboración propia

\section{CPU (Estadísticas de Entrada-Salida)}

\section{CPU sin prueba de carga:}

Media $=1,26 \%$

Desviación estándar $=1,135 \%$

Rango de valores más significativos $=\{1,04 \%-1,49 \%\}$

\section{CPU con prueba de carga:}

Media $=9,18 \%$

Desviación estándar $=6,71 \%$ 
Rango de valores más significativos $=\{8,58 \%-9,76 \%\}$

Los datos generados con y sin prueba de carga (ver Figura 7), indican la variabilidad del porcentaje de utilización de CPU en el servidor, donde los valores mínimos y máximos en cada uno de los casos son extremadamente diferentes y señalan que los intervalos de valores más significativos se alteran. Lo que permite la toma de decisiones a partir del rango confiable de probabilidad de aparición de los mismos. Los valores de las medidas de tendencia central en conjunto con sus medidas de dispersión, determinan el porcentaje más representativo de utilización, además del valor exacto con que tienden alejarse los datos en relación a la muestra con la que se está trabajando.

Por consiguiente, la utilización de CPU se ve afectada por la carga de trabajo que se genera en el sistema en tiempo real, el proceso Squid que se ejecuta al momento de realizar una prueba de carga hacia el servidor y todos los demás procesos del sistema incrementan el porcentaje de utilización del mismo, por lo que los datos generados tienden a crecer en los primeros intervalos de tiempo en que se realiza la transferencia de datos hasta el momento en que vuelva a estabilizarse.

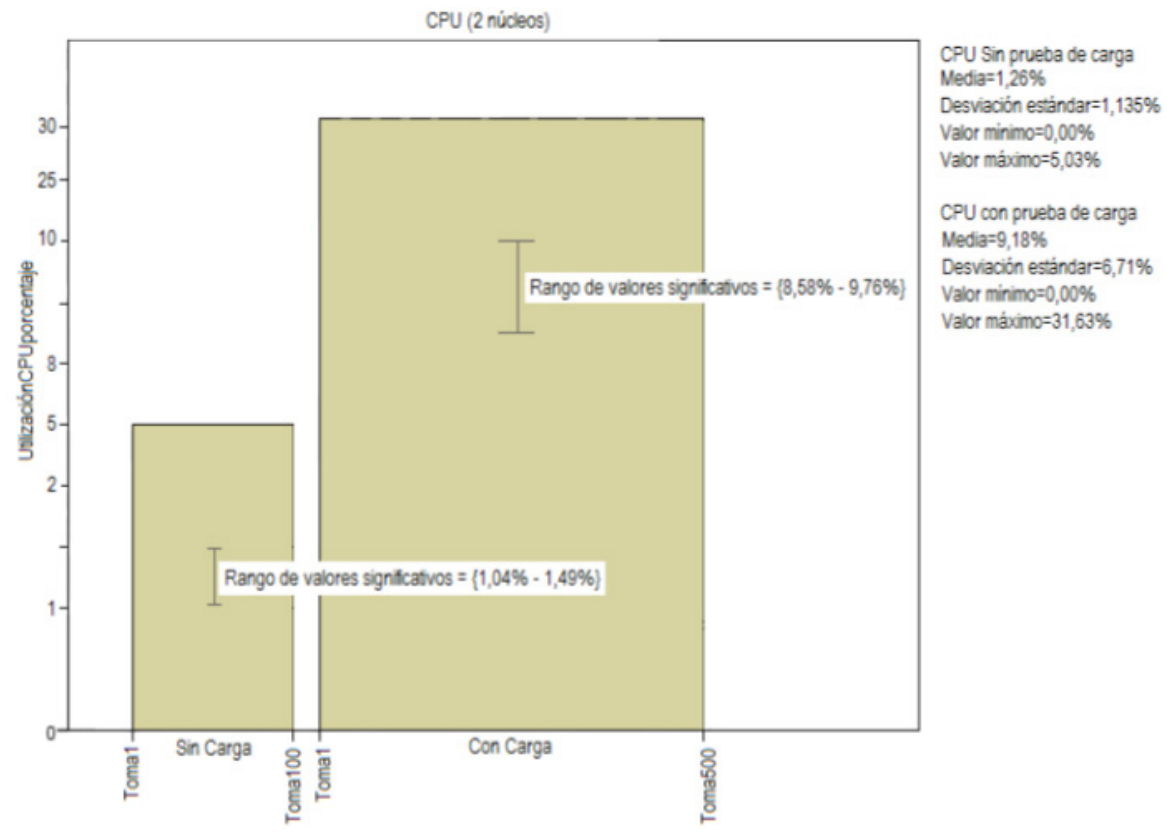

Figura 7. Representación gráfica del rango de valores significativos de CPU con/sin prueba de carga

Fuente: elaboración propia

\section{RED (Estadísticas de Entrada-Salida)}

\section{RED sin prueba de carga:}

Mediana $=0,0000 \mathrm{tps}$

Rango de valores más significativos $=\{2,61 \mathrm{tps}-7,56 \mathrm{tps}\}$ 


\section{RED con prueba de carga:}

Mediana $=0,0000$ tps

Rango de valores más significativos $=\{9,43$ tps $-17,76$ tps $\}$

Los datos generados con y sin prueba de carga (ver Figura 8) indican la variabilidad de las estadísticas de velocidad de transferencia de datos de entrada y salida de RED en el servidor, donde los valores mínimos y máximos en cada uno de los casos son notables y señalan que los intervalos de valores más significativos se alteran. Lo que permite la toma de decisiones a partir del rango confiable de probabilidad de aparición de los mismos. Los valores de las medidas de tendencia central en conjunto con sus medidas de dispersión, determinan el rango de valores muestrales más representativo de transmisiones por segundo de red, además del valor exacto con que tienden alejarse los datos en relación a la muestra con la que se está trabajando.

Por consiguiente, las estadísticas de velocidad de transferencia de datos de entrada y salida de Red, muestran que el proceso Squid genera un gran número de intercambio de datos al momento que se realizan las peticiones de las pruebas de carga, por lo que la cantidad de transmisiones por segundo indican que los accesos múltiples afectan directamente los procesos del servidor mientras estas sigan en espera.

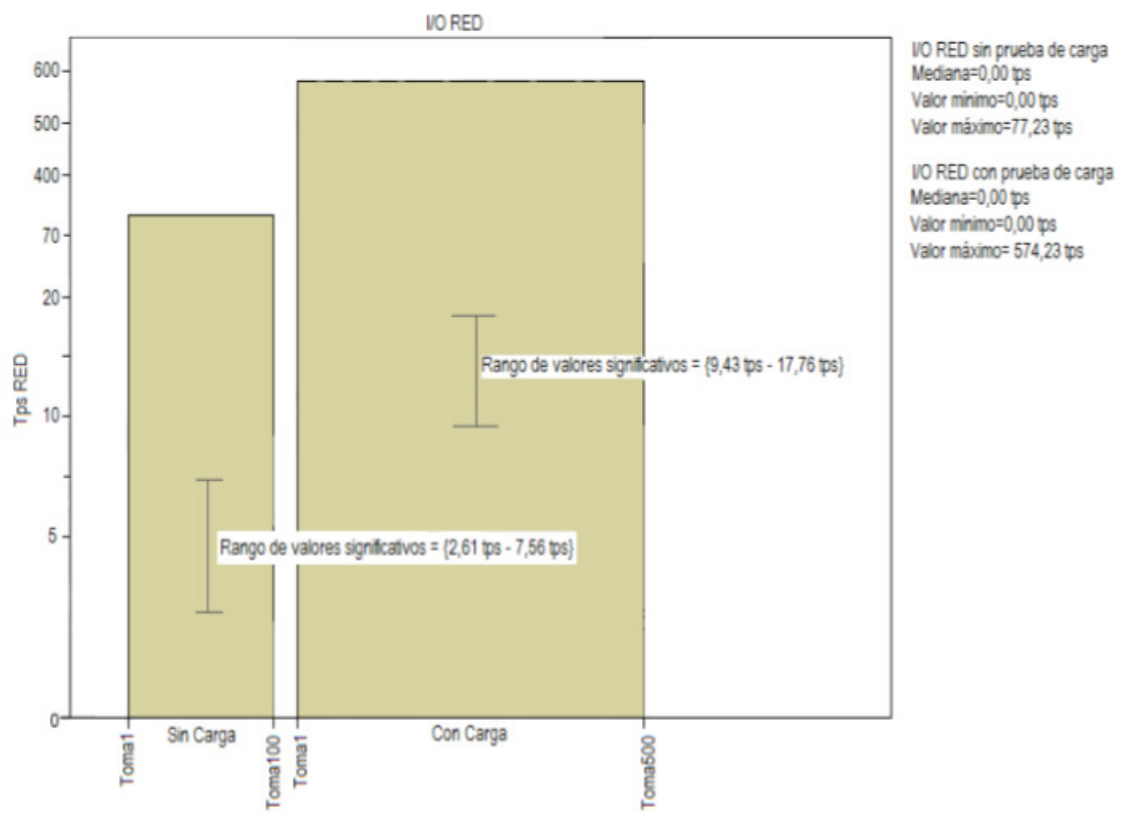

Figura 8. Representación gráfica del rango de valores significativos de I/O RED con/sin prueba de carga Fuente: elaboración propia

\section{RAM (Estadísticas de Entrada-Salida)}

\section{RAM sin prueba de carga:}


Media $=0,93 \mathrm{~Gb}$

Desviación estándar = 0,29 Gb

Rango de valores más significativos $=\{0,87 \mathrm{~Gb}-0,99 \mathrm{~Gb}\}$

\section{RAM con prueba de carga:}

Media $=0,97 \mathrm{~Gb}$

Desviación estándar $=0,49 \mathrm{~Gb}$

Rango de valores más significativos $=\{0,96 \mathrm{~Gb}-1,02 \mathrm{~Gb}\}$

Los datos generados con y sin prueba de carga (ver Figura 9) muestran la variabilidad de los Gb utilizados de memoria RAM en el servidor, distinguiendo los valores mínimos y máximos en cada uno de los casos y señalando los intervalos de valores más significativos. Lo que permite la toma de decisiones a partir del rango confiable de probabilidad de aparición de los mismos. Los valores de las medidas de tendencia central en conjunto con sus medidas de dispersión, determinan la cantidad más representativa de Gb utilizados, además del valor exacto con que tienden alejarse los datos en relación a la muestra con la que se está trabajando.

Por consiguiente, el consumo de memoria Ram, determina que debido a que el proceso squid actúa como un acumulador de memoria caché y normalmente está intercambiando información entre uno o varios clientes e Internet, esta cache conserva copias locales de los datos frecuentemente solicitados y devuelve contenido almacenado cuando es posible, de esa manera, acelera las solicitudes del servicio y reduce el tráfico del ancho de banda.

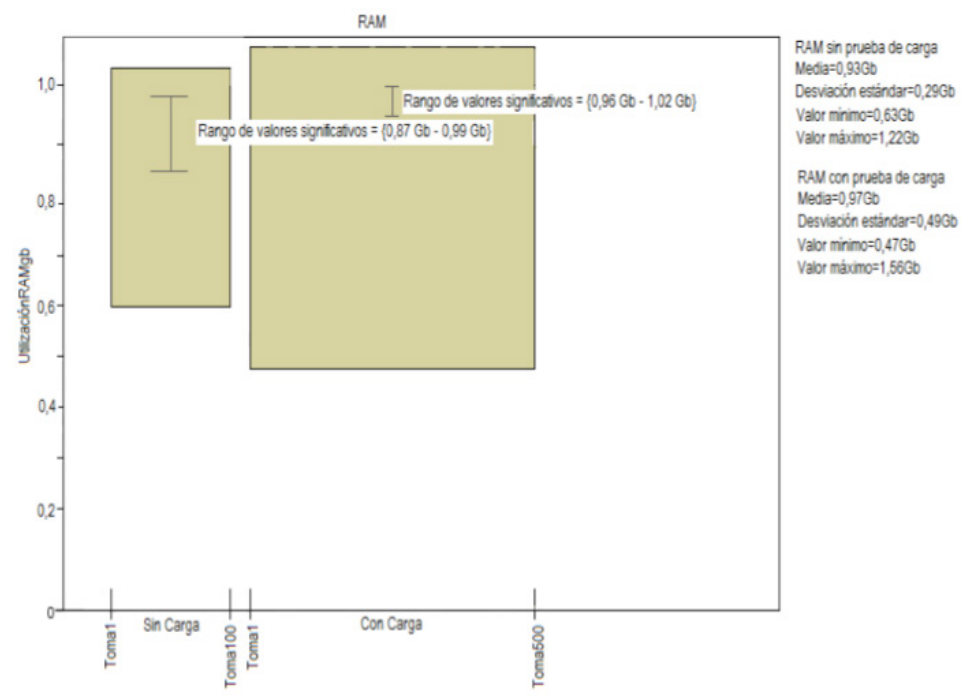

Figura 9. Representación gráfica del rango de valores significativos de RAM con/sin prueba de carga

Fuente: elaboración propia 


\section{Conclusiones}

El modelo funcional para la administración de la red de datos de la DITIC de la UTA se ha establecido en base a modelos de gestión de estándares internacionales, los cuales permiten el manejo óptimo de los recursos con los que se cuenta, y dividen la complejidad de la administración en áreas funcionales que especifican soluciones de gestión.

Las políticas de administración instauradas en base al modelo funcional permiten plantear un sistema de gestión que relacione los requerimientos con la operación correcta de la red, obligando el planteamiento de normas reglamentarias que garanticen la eficiente y efectiva administración del sistema de transmisión de datos.

El desarrollo de la representación técnica de un área funcional muestra que mediante la utilización de los recursos actuales y el tratamiento de las políticas de gestión establecidas, se puede aplicar una metodología de análisis de datos que permita mejorar la calidad de prestación de los servicios que brinda actualmente la DITIC.

El planteamiento de normas reglamentarias definidas por políticas de administración para la red de datos de la DITIC han sido comprobadas por la utilización de aplicaciones y herramientas que permiten la virtualización de un servidor Proxy, para comprobar su resistencia, comportamiento y el rendimiento del mismo, posibilitando la entrega de informes de las actividades del sistema y la generación de datos de administración que permita la toma de decisiones. 


\section{Referencias}

A. S. Foundation. (2013). “Apache JMeter,” 2013. https://jmeter.apache.org/

B. B. T. U. S. T. L. Sti. (2014, febrero). "Uptime robot”.

Dueñas, J. B. (2013). Configuración de squid: Opciones básicas. Publicado el, 23, 48.

Fernández, S. F., Sánchez, J. M. C., Córdoba, A., \& Largo, A. C. (2002). Estadística descriptiva. Esic Editorial.

Galindo, P. P. V., \& Vicente, M. (2012). Estadística para investigadores. Universidad de Salamanca.

Galora Silva, F. J. (2015). Modelo funcional para la administración de la red de datos de la Universidad Técnica de Ambato (Bachelor's thesis, Universidad Técnica de Ambato. Facultad de Ingeniería en Sistemas, Electrónica e Industrial. Carrera de Ingeniería en Electrónica y Comunicaciones).

IBM. (2014). "Software spss".

Montiel, N. A. N. (2005). Diseño y automatización de las políticas de administración de redes de la espol (Doctoral dissertation, Master's thesis, Ingeniería en Computación especialización sistemas tecnológicos ESPOL, Guayaquil-Ecuador).

Naranjo Villacrés, D. R., \& Ortega Tobar, P. E. (2006). Desarrollo de una aplicación gráfica basado en el sistema operativo Linux para el monitoreo y administración del tráfico de datos de redes lan (Bachelor's thesis, QUITO/EPN/2006).

Ruiz, A. M. G., Azkue, G. V., \& Díez, A. G. (2008). Guía práctica para abordar la innovación y su gestión en las empresas de sector de la edificación residencial. Fundación Tekniker.

U. I. de Telecomunicaciones. (2000, febrero). "Recomendación uit-t m.3000".

Vicente, C. A. (2003). Un modelo funcional para la administración de redes. Dirección de telecomunicaciones, Departamento de operación de la red centro de operación de red UNAM (NOC-UNAM). México. 